\title{
A coesão de equipes em cena: Discussão da sua natureza e consequentes
}

Viviane da Mata Barbosa. VMB Consultoria Organizacional. Katia Elizabeth Puente-Palacios. Universidade de Brasília.

\section{Resumo}

Considerando a centralidade da coesão para o bom desempenho de equipes de trabalho, foi realizada uma pesquisa empírica com o objetivo de identificar a estrutura dimensional do fenômeno e elucidar os seus componentes constitutivos, assim como investigar o poder preditivo da coesão em relação à efetividade de equipes. A realização do estudo empírico contou com as respostas de 177 membros de equipes, em uma primeira amostra, e uma segunda amostra composta por 138 participantes. Uma escala de medida da coesão, em língua inglesa, serviu como referência para a elaboração de um instrumento adequado ao contexto brasileiro. Os resultados da análise fatorial exploratória mostraram que a solução unifatorial foi a mais satisfatória e explica aproximadamente $72 \%$ de variância. Análises de padrões de variância dentro e entre grupos revelaram que a medida foca em fenômeno do nível individual e não no nível das equipes (meso). As correlações com os critérios de efetividade mostraram associações significativas e positivas entre as variáveis, mostrando o poder preditivo da medida.

Palavras-chave: processos grupais; efetividade de equipes; satisfação de equipes.

\section{Abstract}

Cohesion of teams on the scene: Discussion of their nature and consequent. Considering the central role that group cohesion plays to obtain a good work team performance, an empirical study was undertaken that sought to identify the dimensional structure of group cohesion revealing its constituent components and to determine the predictive power of group cohesion in relation to team effectiveness. The study obtained the data of 177 team members (sample 1) and 138 participants on a second sample. An instrument measuring cohesion, in English, served as reference for the elaboration of an instrument adapted to the Brazilian context. Results of exploratory factor analysis indicated a unidimensional solution explaining $72 \%$ of the total variance. Analysis of variance within and between groups revealed that the instrument is focusing on the individual level and not on the team level. Correlations with the criteria of effectiveness showed significant and positive associations among the variables, showing the predictive power of the instrument.

Keywords: group processes; effectiveness of teams; team satisfaction.

\section{Resumen}

La cohesión de equipos en escena: Discusión de su naturaleza y consecuentes. Considerando la centralidad de la cohesión para en buen desempeño de los equipos de trabajo, se realizó una investigación empírica con el objetivo de identificar la estructura dimensional del fenómeno, buscando identificar sus componentes estructurantes y verificar el poder de predicción de la cohesión en relación a la efectividad de los equipos. El estudio empírico contó con las respuestas de 177 miembros de equipos (muestra 1) y 138 participantes en una segunda muestra. Una escala de cohesión, en lengua inglesa, sirvió de referencia para la elaboración del instrumento adecuado al contexto brasileño. Los resultados del análisis factorial exploratorio mostraron que la solución más satisfactoria condensa un factor único que explica $72 \%$ de variancia. El análisis de padrones de variancia dentro y entre grupos mostro que la escala foca en un fenómeno individual y no grupal (meso). Las correlaciones con los criterios de efectividad muestran asociaciones significativas positivas entre las variables y revelan el poder predictivo de la medida.

Palabras clave: procesos grupales; efectividad de equipos; satisfacción de equipos. 
Organizações que assumem o cliente como um aspecto central nas estratégias de gestão do negócio, procurando atender melhor e mais rapidamente suas necessidades, têm procurado adotar estruturas matriciais com a formação de equipes multidisciplinares na busca de soluções inovadoras, com menor custo nos processos internos e financeiros. Desse modo, a implementação de equipes no contexto de trabalho como estruturas-chave do desenho empresarial tem sido primordial no alcance de resultados promissores.

Diante da importância da adoção de equipes como estratégia organizacional para alavancagem de resultados, há um interesse crescente, tanto de empresários quanto dos estudiosos da área, pela identificação e compreensão dos fatores e processos que impactam favoravelmente o desempenho dessas unidades (Salas, Reyes, \& McDaniel, 2018). Evidências empíricas demonstram que quando as equipes são devidamente treinadas e as oportunidades são aproveitadas, aumenta a probabilidade de obterem resultados positivos, como criatividade e inovação (Wang \& Rode, 2010), satisfação no trabalho (Puente-Palacios, Seidl, \& Silva, 2008), comprometimento afetivo (Puente-Palacios, Vieira, \& Freire, 2010) e desempenho da equipe (Chi \& Huang, 2014).

Na busca por tal objetivo, os pesquisadores têm centrado seus interesses no estudo das forças sociais e motivacionais que existem entre os membros do grupo. Surge então a hipótese de que estas forças podem promover uma vinculação ou coesão entre os membros e que, quanto mais forte essa ligação, maior a probabilidade de obter bons resultados. Acompanhando tal linha argumentativa, autores da área defendem que se a coesão é forte, o grupo estará mais motivado a coordenar as atividades. Desse modo, a equipe fica apta a ser bem-sucedida no desempenho das tarefas atribuídas (Sanko, 2015).

Portanto, a coesão é uma variável-chave nos modelos sobre efetividade (Jordan, Feild, \& Armenakis, 2002) e tem sido considerada um relevante indicador de viabilidade da equipe, uma vez que associada ao aumento da satisfação da equipe; diminuição da rotatividade e dos comportamentos de cidadania organizacional; e aumento do desempenho (Aoyagi, Cox, \& McGuire, 2008; Forsyth \& Burnette, 2010; Martínez \& Cifre, 2016).

Dada a importância da coesão para o desempenho e satisfação da equipe no contexto de trabaIho, torna-se essencial implementar medidas acuradas que permitam capturar esse construto. Contudo, o desenvolvimento das ferramentas necessárias enfrenta a barreira da falta de concordância teórica entre os pesquisadores da área sobre a definição da natureza do fenômeno e, assim, da sua operacionalização. Este fato traz consequências para a definição da natureza dimensional das medidas propostas (Salas, Grossman, Hughes, \& Coultas, 2015). Alguns pesquisadores da área apontam, ainda, que estudos empíricos têm resultado em conclusões inconsistentes, pois os achados não suportam a visão de que a produtividade do grupo e a coesão estão positivamente relacionadas (Gully, Devine, \& Whitney, 2012). Tal situação faz surgir questionamentos não apenas relacionados à natureza teórica do construto, mas também ao papel que desempenha.

A coesão grupal foi tradicionalmente compreendida como construto unidimensional (Moreira, Montanari, \& Pilatti, 2016) e descrita como as inclinações dos membros do grupo para construir laços sociais que resultam no desejo de permanecerem unidos (Casey-Campbell \& Martens, 2009). Festinger (1950), pesquisador do âmbito da psicologia social, propôs que coesão é um imbricado conjunto de forças que vinculam os membros entre si. A partir dessa visão, coesão é usualmente compreendida nas pesquisas da área como a atração dos membros para o grupo e avaliada a partir de perguntas que indagam o quanto eles gostam uns dos outros ou por quanto tempo eles querem permanecer no grupo (Carless \& De Paola, 2000).

A partir da década de 1990, observa-se uma mudança na compreensão da estrutura interna do fenômeno da coesão. Passa-se a defender sua natureza multidimensional, retomando, desse modo, a proposição inicial de Festinger (1950), para quem a coesão conjuga aspectos como atração interpessoal, engajamento com a tarefa do grupo e sentimento de orgulho coletivo. A defesa dessa abordagem tem como argumento o fato de que, além do papel da atração interpessoal, a coesão envolve também a vinculação ou comprometimento dos membros com a tarefa. E evidências empíricas vêm demonstrando o importante papel da coesão da tarefa e da coesão interpessoal na relação com indicadores como desempenho, satisfação no trabalho e interdependência de tarefas (Gully et al., 2012; Picazo, Gamero, Zorzona, \& Peiró, 2015).

$\mathrm{Na}$ tentativa de elucidar o papel das diversas dimensões da coesão, Mullen e Copper (1994) tiveram uma relevante contribuição ao apontar que a atração interpessoal, o comprometimento com a tarefa e o orgulho do grupo são fatores constitutivos da coesão. 
Buscando esclarecer a pertinência dessas dimensões como componentes da coesão, os autores conduziram uma meta-análise e concluíram que existe relação estatisticamente significativa entre coesão e desempenho, porém o efeito é de escassa magnitude. Em relação ao papel preditivo de cada dimensão, os resultados da pesquisa desses autores demonstraram que o comprometimento com a tarefa do grupo, em oposição à atração interpessoal e orgulho do grupo, foi o único componente que contribuiu de forma independente e significativa para a explicação do desempenho no trabalho. Os resultados, portanto, sugerem que embora tenha sido considerada a existência de três dimensões que compõem a coesão, apenas uma delas teve papel relevante na predição do desempenho.

Aproximadamente dez anos após a pesquisa acima relatada, outro estudo de natureza similar foi conduzido por Beal, Cohen, Burke e McLendon (2003), que também buscou identificar as relações entre coesão e desempenho. Os autores reinvestigaram o papel dos componentes da coesão usando métodos de meta-análise mais robustos e um olhar mais acurado dos diferentes tipos de critérios de desempenho. Ao criticar os achados encontrados no estudo de Mullen e Copper (1994), assumiu-se o pressuposto de que a atração interpessoal, o comprometimento com a tarefa do grupo e o orgulho em relação ao grupo são todos componentes igualmente importantes da coesão da equipe. A partir disso, formularam a hipótese de que os três aspectos deveriam manter relações independentes e significativas com o desempenho do grupo, delimitado nesta pesquisa a partir de dois critérios: desempenho como comportamento e desempenho como resultado. As conclusões encontradas por eles corroboraram a hipótese e revelaram fortes correlações entre coesão e desempenho como comportamento (em oposição a resultado) e indicaram que os três componentes da coesão estão independentemente relacionados aos critérios de desempenho. O conjunto de resultados ora citados revela que não há convergência entre os autores quanto à estrutura multidimensional da coesão, pois embora seja construído um referencial teórico que discute a existência de diversas dimensões, as evidências empíricas são frágeis e deixam dúvidas quanto à pertinência da proposição. Daí os resultados empíricos quanto a essa questão ainda serem inconsistentes.

Embora haja o desencontro dos resultados de pesquisas empíricas relatadas, a relevância da temática para os estudos relativos ao funcionamento de equipes permanece atual, o que justifica a existência de publicações atualizadas para discutir a natureza dimensional do construto ou buscar identificar o papel preditor diferenciado de cada dimensão (Picazo et al., 2015). Estudo conduzido por Salas e colaboradores (2015) aborda a complexidade do fenômeno e discute as divergências entre os pesquisadores em relação à estrutura uni ou multidimensional da coesão. Uma importante contribuição derivada desse trabalho é o destaque das dimensões de coesão mais comumente encontradas na literatura e suas respectivas definições: a) coesão de tarefa é baseada no comprometimento compartilhado e relaciona-se com o alcance das metas e objetivos do grupo; b) coesão social representa uma proximidade e uma atração com o grupo baseada nas relações interpessoais; c) sentimento de pertencimento é o grau no qual os membros do grupo se sentem vinculados uns aos outros; d) orgulho do grupo refere-se à medida na qual os membros demonstram gostar do status ou das ideologias suportadas pelo grupo, ou ainda à importância compartilhada de ser membro do grupo; e e) moral significa o alto grau de lealdade com os colegas de grupo.

Apesar de defender teoricamente a existência de cinco dimensões como elementos constitutivos da coesão, Salas e colaboradores (2015) apontam que as medidas mais efetivas de coesão são aquelas que avaliam os aspectos sociais e de tarefa com ênfase nos aspectos atitudinais e comportamentais. Os autores discutem os resultados do estudo reiterando que a coesão é de fato um construto multidimensional, sendo que coesão social e de tarefa deveriam ser priorizadas como componentes centrais dessa medida. Também afirmam que coesão é um construto multinível, o que significa dizer que é legítimo defender a sua ocorrência no nível individual e no nível coletivo, e que por operarem mais consistentemente no nível da equipe, as pesquisas deveriam focar no nível meso. Por fim, reforçam a ideia de que embora um progresso significativo tenha ocorrido ao longo dos anos, é necessário ainda um impulso teórico e a construção de medidas mais robustas, precisas e adequadas.

De modo a esclarecer questões pertinentes com relação ao construto coesão de equipes e seus efeitos no desempenho das equipes, recente estudo realizado por Picazo et al. (2015), teve como objetivo testar as relações entre coesão e satisfação em equipes de trabalho, assumindo a visão bidimensional (interpessoal e tarefas) proposta por Salas et al. (2015). Assim, analisaram a relação da coesão da tarefa e a coesão 
interpessoal em relação a um critério afetivo de efetividade, ou seja, a satisfação dos membros com sua equipe de trabalho. Os autores apontam que, embora a efetividade da equipe seja geralmente examinada em termos de desempenho, outros indicadores são pertinentes e com eles se coadunam ao conceito prevalente de efetividade da equipe proposto por Hackman (1987), no qual são condensados indicadores de desempenho, indicadores afetivos e de viabilidade da equipe. Ao revisar a literatura da área, constata-se que diversos autores têm vinculado a coesão da equipe a resultados afetivos (Kozlowski \& Ilgen, 2006).

Diante do exposto e das lacunas antes referidas, o objetivo do presente artigo é identificar a estrutura dimensional da coesão grupal, visando elucidar os seus componentes constitutivos, mediante a realização de uma pesquisa empírica que demonstre o seu poder preditivo em relação à efetividade de equipes. As etapas de execução da fase empírica deste estudo estão relatadas na seção que segue.

\section{Método}

\section{Participantes}

A amostra principal para a realização desta pesquisa contemplou 177 profissionais integrantes das equipes de Saúde da Família (eSF), da Secretaria da Saúde do Governo do Distrito Federal, organizados em 38 equipes. Esse quantitativo representa 100\% do total de questionários aplicados (não houve questionários devolvidos em branco) e se justifica por ter sido coleta presencial, no local de trabalho das equipes, realizada durante reuniões semanais. Os dados demográficos dessa amostra revelaram que uma elevada porcentagem dos respondentes é do sexo feminino $(73,4 \%)$ e a idade média é de $39,4 \%(D P=8)$. As equipes foram compostas por profissionais que ocupavam as funções de médico (14,1\%), dentista (8\%), técnico de enfermagem (26,6\%), técnico de higiene bucal - THD $(3,4 \%)$. A maioria é composta por agentes comunitários de saúde - ACS (48,6\%). A quantidade de integrantes por equipe variou de 4 a 11 pessoas. Ainda houve uma segunda amostra utilizada para fins de verificação do comportamento da medida, composta por 138 respondentes de instituição pública, organizados em 42 equipes. A grande maioria deles era do sexo masculino $(62,3 \%)$ e adulto $(36,6$ anos, $D P=11,6)$. Esta coleta ocorreu no mês de agosto de 2016 e os mesmos procedimentos foram adotados para a aplicação das medidas.
Ambas as amostras foram de conveniência, portanto, a coleta de dados se estendeu temporalmente tanto quanto os respondentes se dispunham a participar.

\section{Procedimento}

Os questionários foram aplicados em formato impresso durante as reuniões das equipes de trabalho ocorridas entre os meses de fevereiro e agosto de 2015. Antes da aplicação do instrumento, a pesquisadora conduziu uma exposição geral a respeito dos objetivos do estudo e, após essa introdução, o questionário foi entregue para cada integrante, momento em que era destacado o caráter voluntário da participação na pesquisa. Desse modo, os princípios éticos que norteiam as pesquisas com seres humanos foram respeitados.

\section{Instrumentos}

A efetividade da equipe foi avaliada por meio de dois critérios. O primeiro deles, desempenho julgamental, foi avaliado mediante a escala de Puente-Palacios, Martins e Palumbo (2016), composta por nove afirmativas de desempenho apresentados na forma de itens respondidos em escala do tipo Likert, na qual 1 corresponde a "discordo totalmente" e 5 a "concordo totalmente", com Alpha de Cronbach de 0,93 e correlação item-total de 0,76 . Os itens desta medida se organizam em solução unifatorial. O segundo critério foi a satisfação com a equipe de trabalho, capturada pelo instrumento de satisfação com equipes (Puente-Palacios \& Borges-Andrade, 2005). Essa medida possui cinco itens, agrupados em fator único, respondidos em escala do tipo Likert de cinco pontos, na qual 1 corresponde a "discordo totalmente" e 5 a "concordo totalmente" $(a=0,88 ; r$ item-total $=0,72$ ).

A escala Group Cohesiveness (Langfred, 1998) serviu como base para a elaboração de um instrumento traduzido para a língua portuguesa. A tradução do inglês para o português foi conduzida com o uso da técnica de tradução-retradução (Hambleton, 1994). Paralelamente foram observadas as orientações dadas por Borsa, Damásio e Banderia (2012) que recomendam a realização de duas traduções independentes para o português, seguidas da comparação e análise das versões. O conteúdo final dos itens da medida, segundo esses autores, deve representar uma síntese das traduções, conforme avaliada por experts da área, levando em consideração também o perfil do público a que será aplicado. Seguindo essas orientações, as duas versões em inglês foram comparadas entre si e com o original, o que levou a acertos finais na escala em português. 
A versão final foi revisada por uma especialista na área de psicologia organizacional e ainda foi verificada a sua adequação semântica junto a trabalhadores com perfil profissional similar ao do público-alvo. A medida original está composta por seis itens respondidos em escala do tipo Likert de concordância de cinco pontos. A organização desses itens em fatores tem se dado em dois fatores que separam a coesão de tarefas e social. No caso da segunda amostra, a medida de coesão aplicada foi a resultante após as análises realizadas e descritas na seção Resultados deste artigo. Assim, a escala efetivamente respondida continha apenas três itens de coesão. A medida de desempenho foi exatamente a mesma aplicada à amostra principal do estudo.

\section{Análise de Dados}

Tendo em vista os objetivos deste estudo, os procedimentos analíticos adotados foram relacionados à análise da estrutura interna da medida de coesão, mediante a realização de uma análise fatorial exploratória (AFE). Investigou-se o número adequado de fatores a reter e a adequação da solução por meio da verificação dos índices de consistência interna dos fatores. A seguir, considerando que os dados foram coletados no nível individual, porém conjecturando que a coesão pode ser compreendida como atributo coletivo, verificou-se a magnitude da semelhança das respostas dadas pelos membros de cada equipe, tendo como referência o cálculo do índice de desvios (ADMd). Em seguida, buscou-se evidências de variância entre equipes calculando, para tanto, a magnitude da correlação intraclasse (ICC), assim como a diferença das médias pelo uso de uma Anova (one-way). Por fim, buscando analisar as relações entre coesão e os critérios de efetividade adotados neste estudo, foi conduzida uma análise de correlação bivariada. Os resultados da aplicação desse conjunto de estratégias analíticas são descritos a seguir. Todas as análises foram realizadas mediante o uso do pacote de análises estatísticas SPSS, versão 21.

\section{Resultados}

Buscando investigar a pertinência de realizar as análises aderentes ao objetivo do estudo foi verificada, em primeira instância, a adequação do tamanho da amostra para a análise fatorial exploratória. Hair, Anderson, Tatham e Black (2009), recomendam que haja, pelo menos, cinco respondentes por item inserido nas análises. Pasquali (2010), por sua vez, defende que são desejáveis 10 respondentes por item da medida.
Considerando que a amostra principal do estudo conta com 177 respondentes, conclui-se que o tamanho da amostra é compatível com as análises a ser realizadas. Em relação à estrutura subjacente à medida utilizada, em um primeiro momento foi investigada a fatorabilidade da matriz de dados com a observação de quatro critérios: (a) o cálculo da magnitude do determinante da matriz; (b) o KMO de adequação da amostra; (c) o teste de Esfericidade de Bartlett; e (d) a inspeção da matriz de correlações. Os valores obtidos na verificação desses critérios foram aceitáveis, tanto para o determinante da matriz de dados $(0,23)$, quanto para o $\operatorname{KMO}(0,66)$, assim como para o teste de esfericidade $(p>0,01)$, indicando que a matriz pode ser reduzida a fatores. $A$ inspeção da matriz de dados revelou diversas associações significativas e positivas entre alguns itens da medida. Contudo, observou-se que o item 6 ("os membros da minha equipe fazem coisas juntos quando estão de folga") não apresentou correlação significativa com qualquer um dos outros itens da medida. Já o item 5 ("tenho sentido desejo de mudar para outra equipe") revelou estar associado apenas com um dos itens da escala. Esse conjunto de dados mostrou que era pertinente ir adiante na tarefa de investigação da estrutura fatorial da medida, mas ao mesmo tempo relevou comportamentos anômalos de alguns itens, os quais deveriam ter o seu comportamento analisado cuidadosamente.

A seguinte fase da análise consistiu na definição do número adequado de fatores a reter. Para tanto, observando os valores dos eigenvalues encontra-se que a matriz de dados suporta até dois fatores e, desde uma perspectiva teórica, a escala original utilizada como base da medida considerada neste estudo possui uma estrutura bidimensional que engloba as dimensões denominadas coesão de tarefas e coesão interpessoal. Assim, investigou-se a pertinência de reter dois fatores (rotação Promax).

A análise da matriz fatorial resultante da extração de dois fatores mostrou inadequação dos resultados, pois dos seis itens da medida, o número 4 ("meus companheiros não me ajudam quando eu tenho um problema") não trouxe contribuições significativas para qualquer um dos fatores, por apresentar carga inferior a 0,30 . Esse valor de carga fatorial foi adotado seguindo as orientações de Tabachnick e Fidel (2007) que destacam que esse $(0,30)$ é o valor mínimo de contribuição (carga fatorial) de um item para a mensuração do construto latente. Tendo em vista esse achado insatisfatório, investigou-se a pertinência de reter apenas um 
fator da matriz de dados e essa solução mostrou-se adequada, embora resultasse na perda adicional de dois itens. Portanto, conclui-se que a solução fatorial mais satisfatória para a escala em análise é composta por três itens, organizados em uma estrutura unifatorial, sendo que dos seis originais, dois deles (excluídos) já tinham mostrado comportamento anômalo no momento da inspeção da matriz de correlações (itens 5 e 6).

Quanto ao desempenho dos itens que efetivamente mostram-se como constitutivos da medida observam-se cargas fatoriais entre 0,69 e 0,86; e, organizados em estrutura unifatorial, capturam um total de variância explicada que ascende a $72 \%$, conforme pode ser visualizado na Tabela 1. Dos itens da medida, aquele que melhor representa o construto subjacente é o terceiro que indaga sobre o fato do respondente confiar nos seus colegas de equipe ao enfrentar problemas ou dificuldades. A pertinência da decisão tomada quanto à extração de um fator único também esteve sustentada na avaliação da confiabilidade interna desse fator ( $a=0,80 ; r$ item-total $=0,73)$, com resultados considerados satisfatórios.

Tabela 1. Resultados da Análise Fatorial Exploratória da Escala de Coesão da Equipe de Trabalho.

\begin{tabular}{lc}
\hline \multicolumn{1}{c}{ Enunciado do item } & Fator 1 \\
\hline $\begin{array}{lc}\text { 1. Confio em meus colegas do grupo para me ajudar e apoiar } \\
\text { quando estou tendo dificuldades. }\end{array}$ & 0,86 \\
$\begin{array}{l}\text { 2. Conto com os outros membros da equipe para conseguir } \\
\text { completar minha tarefa. }\end{array}$ & 0,75 \\
$\begin{array}{l}\text { 3. Os demais membros da equipe são preparados para dar } \\
\text { conselhos e me ajudar em minhas tarefas. }\end{array}$ & 0,69 \\
\% Variância explicada & $72 \%$ \\
No de Itens & 3 \\
Alpha de Cronbach & 0,80 \\
Média da correlação item-total & 0,73 \\
\hline
\end{tabular}

Buscando obter evidências adicionais da pertinência das decisões tomadas no sentido de eliminar três dos seis itens originais da medida, foi realizada uma nova AFE na segunda amostra composta pelas respostas de 138 trabalhadores. Os mesmos procedimentos adotados na realização dessa análise na amostra principal foram seguidos e os resultados obtidos revelaram que todos os três itens tiveram cargas fatoriais acima do valor mínimo $(0,30)$ e oscilaram entre 0,70 e 0,84. A confiabilidade do fator único obtido a partir do cálculo do alfa de Cronbach $(0,80)$ e da magnitude da correlação item total $(0,65)$ também foi satisfatória e se constitui em indicador adicional da pertinência da decisão tomada quanto à redução da medida de seis pra três itens. Nesta amostra, a solução unifatorial retendo os três itens permitiu captu$\operatorname{rar} 73,07 \%$ da variância do fenômeno.

Uma vez definida a estrutura unidimensional do instrumento de coesão, o passo natural seguinte seria verificar a existência de associações entre essa variável e critérios de efetividade da equipe. Entretanto, ponderou-se quanto ao nível apropriado de execução dessas análises. Isto é, questionou-se a pertinência de focar na percepção do indivíduo sobre a coesão existente na sua equipe, tendo em vista que o fenômeno em si é compreendido como atributo coletivo, o que exige a criação de um escore grupal. Seguindo as orientações de Martínez e Cifre (2016), buscamos verificar a pertinência de fazer emersão analisando a existência de diferenças significativas entre as equipes da amostra, de sorte a evidenciar a adequação de tratar a coesão um fenômeno do nível meso. A indagação ora referida foi realizada mediante o cálculo de uma Anova (one-way) e do coeficiente de correlação intraclasse (ICC). Os resultados na Anova não foram significativos $(p=0,4)$ e a magnitude do ICC foi de escasso valor $(0,014)$, o que demonstra não existir, na amostra pesquisada, diferenças significativas entre as equipes no que diz respeito ao nível de coesão percebido. Portanto, não pode ser tratada como propriedade grupal.

Esses achados sustentaram a decisão de realizar as análises seguintes no nível individual. Ou seja, buscou-se verificar a existência de relações entre coesão percebida na equipe e a satisfação individualmente relatada com a experiência de trabalho coletivo; e entre coesão e avaliação do desempenho da equipe feita pelos membros. Uma vez que essas duas variáveis constituíam indagações relacionadas à equipe, também se investigou a pertinência de serem tratadas c omo atributos individuais. Para tanto, foram realizadas análises de variância e correlações intraclasse. No caso de ambas as variáveis, os resultados obtidos mostraram tratar-se de variáveis do nível individual, uma vez que os resultados das Anovas não foram significativos (Desempenho: $p=0,25$; Satisfação: $p=0,27$ ) e a magnitude do ICC foi escassa (Desempenho = 0,046; Satisfação = 0,046).

A seguir foram realizadas correlações bivariadas com os dados da amostra principal entre coesão e satisfação e entre coesão e desempenho, ambas no nível individual. Os resultados obtidos revelaram associações significativas e positivas entre essas variáveis, contudo, o maior efeito foi identificado ao associar coesão e desempenho $(r=0,5 ; p<0,01)$ quando comparados 
com o observado entre coesão e satisfação $(r=0,37$; $p<0,01)$. Tais valores significam que existe quase 0 dobro de variância compartilhada entre a percepção do membro sobre a coesão existente na sua equipe e a avaliação que ele faz sobre o desempenho coletivamente demonstrado (25\%), do que a compartilhada entre coesão e satisfação com a experiência de trabaIho feito em conjunto (13,6\%).

Também quanto a estas análises buscou-se investigar de maneira mais robusta as associações ora relatadas. Desse modo, análises similares (de correlação) foram realizadas na segunda amostra antes mencionada, na qual foram levantados dados de coesão apenas com os três itens retidos no estudo objeto deste manuscrito, além de dados relativos a desempenho da equipe, avaliados mediante o uso da mesma escala (nove itens e solução unifatorial, $a=0,94$, correlação item total $=0,76)$. Os resultados revelaram correlação positiva e significativa entre os construtos. Assim, a coesão relatada pelos membros das equipes está positivamente associada às percepções que eles possuem sobre o desempenho das suas unidades de desempenho $(r=0,36 ; p<0,05)$. Desse modo, conta-se com evidências adicionais da validade externa da medida de coesão composta por três itens. As implicações e derivações do estudo descrito e das escolhas metodológicas realizadas são apresentadas na seguinte seção e analisadas sob a perspectiva das teorias da área.

\section{Discussão}

Este estudo propõe identificar a estrutura dimensional da coesão de equipes e elucidar os seus componentes constitutivos por meio de uma pesquisa empírica que demonstre seu poder preditivo em relação ao desempenho e à satisfação de equipes de trabalho obtendo, desse modo, evidências da validade externa da medida. Os resultados da análise fatorial da medida de coesão apontaram para uma estrutura unifatorial organizada em três itens, dos seis originais. São estes que carregam fortemente características relacionadas à coesão de tarefa, ou seja, itens com conteúdo focado nos esforços coletivos do grupo para alcançar as metas comuns.

Embora no presente estudo os itens relativos à dimensão social da coesão não tenham sido retidos, os resultados empíricos obtidos são um importante avanço, uma vez que há apenas uma medida de coesão de equipes com evidências de validade divulgadas nas publicações científicas da literatura brasileira, elaborada por Moreira et al. (2016). Ainda, a escala apresentada neste estudo possui indicadores psicométricos robustos o suficiente para sua devida e correta utilização.

A relevância dessa medida é reforçada com os resultados deste estudo, que mostram relação positiva e significativa entre coesão e efetividade de equipe, mais especificamente com desempenho e satisfação, sendo consistente com discussões anteriores que apontam que equipes com maior coesão tendem a ser mais efetivas quando comparadas com outras menos coesas. Importante destacar a coesão, pois $25 \%$ da variância em relação à percepção de desempenho reitera conclusões prévias de estudos nos quais a coesão de tarefa foi mais fortemente associada ao desempenho da equipe do que à coesão interpessoal (Carless \& De Paola, 2000).

Outro achado relevante é a relação da coesão grupal com a satisfação da equipe, o que faz possível afirmar que uma equipe coesa tende a estabelecer relações sociais entre os membros e promover um sentimento positivo de cumprimento de expectativas. Pondera-se quanto ao fato de a equipe de trabalho ser o cenário que permite ao sujeito satisfazer as suas necessidades pessoais e ter visão favorável sobre a experiência de trabalho compartilhado.

Apesar dos achados do presente estudo apontar contribuições relevantes na compreensão da natureza da coesão de equipes e do seu aspecto dimensional, assim como demonstrar sua relevância na compreensão dos processos grupais para melhor gestão das equipes de trabalho e, consequentemente, o alcance de melhores resultados, é importante considerar a complexidade do fenômeno em questão, o que requer a ampliação de estudos sobre este tema e a realização de pesquisas dentro do contexto brasileiro.

Outro aspecto que merece ser contemplado é o fato dos resultados empíricos divulgados nas publicações da literatura internacional mais recentes apontarem para a coesão de equipes como construto multidimensional, conforme discutido por Salas e colaboradores (2015). Mais especificamente, os autores propõem que coesão social e de tarefa devem ser priorizadas como componentes centrais dessa medida. E, ainda, no estudo conduzido por Picazo et al. (2015) foi utilizada uma medida com duas dimensões: coesão de tarefas e coesão interpessoal. Assim como na pesquisa de Sanko (2015), cujo instrumento de coesão de equipes foi construído a partir de escalas distintas compondo as seguintes dimensões: a) integração do 
grupo de tarefa; b) valor instrumental; c) atração entre os membros; d) compatibilidade. Essas informações apontam para o avanço na compreensão da medida e seu caráter multifatorial.

Quanto à associação entre coesão e desempenho das equipes, assim como coesão e satisfação das equipes analisada na presente pesquisa, os resultados confirmam os achados de outras pesquisas na área. Por exemplo, os resultados da pesquisa longitudinal conduzida por Picazo et al. (2015), demonstraram que a coesão de tarefas emergiu mais fortemente do que a coesão interpessoal durante os primeiros estágios do trabalho desenvolvido pelas equipes de projeto. E que, ao longo do tempo, a relação entre coesão interpessoal e satisfação com equipe foi mediada pela coesão de tarefas. Ainda, Sanko (2015) corroborou a hipótese da coesão de equipes estar associada ao desempenho da equipe. E em estudo multinível conduzido por Martínez e Cifre (2016), os resultados sugerem que a satisfação da equipe tem antecedentes nas variáveis individuais e grupais.

Diante do exposto, embora a coesão de tarefa e a coesão interpessoal sejam considerados relevantes processos interpessoais na constituição das equipes de trabalho, cabe destacar os argumentos de Marks, Mathieu e Zaccaro (2001), que defendem ser a coesão de tarefa um processo motivacional mais diretamente orientado para o alto desempenho. A coesão interpessoal pode atuar como antecedente da coesão de tarefas, uma vez que promove os vínculos afetivos e gera maior comprometimento dos membros para a realização de suas tarefas.

Os resultados sugerem que a força da relação entre coesão e desempenho pode estar substancialmente determinada pelo comprometimento do grupo no cumprimento das tarefas solicitadas, gerando maior necessidade de coordenação, comunicação e monitoramento da performance entre os membros do grupo, o que por sua vez aumenta a percepção de um desempenho mais efetivo. Embora a coesão de tarefas, neste estudo, tenha relação positiva também com a satisfação da equipe, considera-se que há outros aspectos da coesão não avaliados no presente estudo que podem ter maior poder explicativo nessa relação, como, por exemplo, a coesão interpessoal que, conforme abordado por Picazo et al. (2015), tende a assumir papel relevante na satisfação quando analisada em conjunto com a coesão de tarefas.

Diante do exposto, é importante reforçar a necessidade do desenvolvimento de novas escalas adaptadas ao Brasil e a realização de outros estudos empíricos, uma vez que as evidências sugerem que a coesão de equipes é um construto multidimensional. Uma importante contribuição nesse sentido foi a adaptação do instrumento de pesquisa norte-americano Multidimensional Sport Cohesion Instrument (MSCl), utilizado para mensurar a coesão de equipes na área esportiva, para a realidade empresarial, acadêmica e esportiva no Brasil, com quatro dimensões, 22 itens e alpha de Cronbach superior $a=0,70$.

O presente estudo responde a uma série de agendas de pesquisa do campo internacional e traz como perspectiva o avanço na discussão da natureza e do papel da coesão para a efetividade das equipes. Além disso, contribui para o desenvolvimento de uma medida coerente com a literatura, conforme sugerido por Salas e seus colaboradores (2015). Os pesquisadores interessados na compreensão das equipes de trabaIho e nos processos que podem alavancar seus resultados têm um grande desafio pela frente. Evidencia-se assim a importância de tornar isso uma prioridade.

A pesquisa ora relatada também permite derivar algumas contribuições práticas. Por exemplo, os gestores devem estar atentos aos vínculos estabelecidos entre os membros das equipes que comandam. Contudo, contrariando ao que o senso comum sugere, esses vínculos não se reduzem a laços de amizade. O reconhecimento do outro como um par que colabora e coopera para a realização das demandas de trabalho postas (coesão de tarefas) constitui um importante preditor de resultados favoráveis ao funcionamento das equipes. Assim, o gestor deve implementar ações ou práticas que permitam tornar mais evidente a contribuição de cada integrante e a vinculação dessas contribuições para o alcance da meta coletiva. Tais práticas podem fortalecer a coesão de tarefas, fenômeno que esta pesquisa associa de maneira significativa tanto à satisfação quanto ao desempenho.

Quanto às limitações do estudo, é necessário reconhecer que elas derivam do fato de ser um estudo de corte transversal, de ter somente medidas de autorelato e das duas amostras serem da mesma região do País. Assim, cautela na leitura dos achados se faz necessária. Por fim, reconhece-se a necessidade de ampliação de pesquisas sobre o tema, pois um único estudo não oferece evidências suficientes sobre o comportamento e a natureza do fenômeno, considerando que a medida pode sofrer alterações dependendo de atributos da amostra de respondentes. Este é um primeiro passo promissor para o desenvolvimento do campo e 
para a melhor compreensão desse fenômeno como importante processo grupal na condução de resultados de equipe mais efetivos no contexto de trabalho.

\section{Referências}

Aoyagi, M. W., Cox, R. H., \& McGuire, R. T. (2008). Organizational citizenship behavior in sport: relationships with leadership, team cohesion, and athlete satisfaction. Journal of Applied Psychology, 20, 25-41. doi: 10.1080/10413200701784858

Beal, D., Cohen, R., Burke, M., \& McLendon, C. (2003). Cohesion and performance in groups: A meta-analytic clarification of construct relations. Journal of Applied Psychology, 88(6), 989-1004. doi: 10.1037/0021-9010.88.6.989

Borsa, J., Damásio, B., \& Banderia, D. (2012). Adaptação e validação de instrumentos psicológicos entre culturas: algumas considerações. Paidéia, 22, 423-432. doi: 10.1590/S0103-863X2012000300014

Casey-Campbell, M., \& Martens, M. (2009). Sticking it all together: A critical assessement of the group cohesion - performance literature. International Journal of Management Reviews, 11(2), 223-246. doi: 10.1111/j.1468-2370.2008.00239.x

Carless, S., \& De Paola, C. (2000). The measurement of cohesion in work teams. Small Group Research, 31, 71-88. doi: 10.1177\%2F104649640003100104

Chi, N., \& Huang, J. (2014). Mechanisms linking transformational leadership and team performance: The mediating roles of team goal orientation and group affective tone. Group \& Organization Management, 39(3), 300-325. doi: 10.1177\%2F1059601114522321

Festinger, L. (1950). Informal Social Communication. Psychological Review, 57, 271-284. doi: 10.1037/h0056932

Forsyth, D. R., \& Burnette, J. (2010). Group processes. In R. F. Baumeister \& E. J. Finkel (Orgs.), Advanced social psychology: The state of the science (pp. 495-534). New York: Oxford University Press

Gully, S. M., Devine, D. J., \& Whitney, D. J. (2012). A meta-analysis of cohesion and performance: Effects of level of analysis and task interdependence. Small Group Research, 43(6), 702-725. doi: 10.1177/1046496412468069

Hackman, J. R. (1987). The design of work teams. In J. W. Lorsch (Org.), Handbook of organizational behavior (pp. 315-342). Englewood Cliffs, NJ: Prentice-Hall.

Hair, J. F., Anderson, R. E., Tatham, R. L., \& Black, W.C. (2009). Análise multivariada de dados. Porto Alegre: Bookman.

Jordan, M. H., Field, H. S., \& Armenakis, A. A. (2002). The relationship of group process variables and team performance: A team-level analysis in a field setting. Small Group Research, 33, 121-150. doi: $10.1177 / 104649640203300104$

Kozlowski, S. W. J., \& Ilgen, D. R. (2006). Enhancing the effectiveness of work groups and teams. Psychological Science in the Public Interest, 7, 77-124. doi: 10.1111\%2Fj.1529-1006.2006.00030.x

Langfred, C. (1998). Is group cohesiveness a double-edged sword? An investigation of the effects of cohesiveness on performance. Small Group Research, 29, 124-143. doi: 10.1177/1046496498291005

Hambleton, R. K. (1994). Guidelines for adapting educational and psychological tests: A progress report. European Journal of Psychological Assessment, 10(3), 229-244.
Marks, M. A., Mathieu, J. E., \& Zaccaro, S. J. (2001). A temporally based framework and taxonomy of team process. Academy of Management Review, 26(3), 356-376. doi: 10.5465/amr.2001.4845785

Martínez, I., \& Cifre, E. (2016). Individual and group antecedents of job satisfaction: A one-lab multilevel study. Anales de Psicología, 32(2), 565-570. doi: 10.6018/analesps.32.2.216801

Moreira, S., Montanari, R., \& Pilatti, L. (2016). Coesão de equipes: a adaptação do instrumento de pesquisa norte-americano Multidimensional Sport Cohesion Instrument para a realidade empresarial e esportiva no Brasil. Gestão \& Produção, 23(4), 662-675. doi: $10.1590 / 0104-530 \times 961-16$

Mullen, B., \& Copper, C. (1994). The relation between group cohesiveness and performance: An integration. Psychological Bulletin, 115, 210-227. doi: 10.1037/0033-2909.115.2.210

Pasquali, L. (2010). Instrumentação psicológica. Porto Alegre: Artmed.

Picazo, C., Gamero, N., Zorzona, A, \& Peiró, J. M. (2015). Testing relations between group cohesion and satisfaction in Project teams: A cross-level and cross-lagged approach. European Journal of Work and Organizational Psychology, 24(2), 297-307. doi: 10.1080/1359432X.2014.894979

Puente-Palacios, K. E., \& Borges-Andrade, J. E. (2005). O efeito da interdependência na satisfação de equipes de trabalho: um estudo multinível. Revista de Administração Contemporânea, 9(3), 57-78. doi: 10.1590/S1415-65552005000300004

Puente-Palacios, K. E., Martins. M. C., \& Palumbo, S. (2016). Desempenho de equipes: evidências de validade de uma medida. PSICO-USF, 21(3), 513-525. doi: 10.1590/1413-82712016210306

Puente-Palacios, K. E., Seidl. J., \& Silva, R. A. (2008). Ser ou parecer diferente: o papel da diversidade na satisfação de equipes de trabalho. Psicologia: Organizações e Trabalho, 8(2), 79-97. Recuperado de http://pepsic.bvsalud.org/scielo.php?script=sci_ arttext\&pid=S1984-66572008000200005\&Ing=pt\&tIng=pt

Puente-Palacios, K. E., Vieira, R. A., \& Freire, R. A. (2010). O impacto do clima no comprometimento afetivo em equipes de trabalho. Avaliação Psicológica, 9(2), 311-322. Recuperado de http://pepsic.bvsalud.org/scielo.php?script=sci_arttext \&pid=S1677-04712010000200015\&lng=pt\&tIng=pt

Salas, E., Cooke, N., \& Rosen, M. (2008). On teams, teamwork and team performance: Discoveries and developments. Human Factors, 50(3), 540-547. doi: 10.1518/001872008X288457

Salas, E., Grossman, R., Hughes, A., \& Coultas, C. (2015). Measuring team cohesion: Observations from the Science. Human Factors, 57(3), 365-374. doi: 10.1177/0018720815578267

Salas, E. Reyes, D., \& McDaniel. S. (2018). The science the teamwork: Progress, reflections, and the road ahead. American Pshicological Association, 73(4), 593-600. doi: 10.1037/amp0000334

Sanko, J. (2015). Exploring the cohesion-performance relationship in inter-professional health care teams (Dissertação de Doutorado). Recuperado de https://scholarlyrepository.miami.edu/cgi/viewcontent. cgi?article=2387\&context=0a_dissertations.

Tabachnick, B. G., \& Fidell, L. S. (2007). Using multivariate statistics (5 ed.). Boston, MA: Allyn \& Bacon.

Wang, P., \& Rode, J. (2010). Transformational leadership and follower creativity: The moderating effects of identification with leader and organizational climate. Human Relations, 63(8), 1105-1128. doi: $10.1177 / 0018726709354132$ 
Viviane da Mata Barbosa, Mestre em Psicologia Social, Organizacional e do Trabalho pela Universidade de Brasília (UnB), é fundadora e consultora da empresa VMB Consultoria Organizacional. Telefone para contato: (61) 981347482.

E-mail: viviane@vivianedamata.com.br

Katia Elizabeth Puente-Palacios, Doutora em Psicologia Social, Organizacional e do Trabalho pela Universidade de Brasília (UnB) e Pós-doutorado na Universidade de Valencia (UVA - Espanha), é

Professora da Universidade de Brasília (UnB).

E-mail: kep.palacios@gmail.com

Recebido em 24.Jan.17

Revisado em 23.Jul.17

Aceito em 19.Set.18 